\title{
COLLICULAR ENSEMBLE CODING OF SACCADES BASED ON VECTOR SUMMATION
}

\author{
J. A. M. Van Gisbergen, ${ }^{*}$ A. J. Van Opstal and A. A. M. TAX \\ Department of Medical Physics and Biophysics, University of Nijmegen, P.O. Box 9101, 6500 HB \\ Nijmegen, The Netherlands
}

\begin{abstract}
The superior colliculus in the monkey contains a topographically organized representation of the target in its upper layers and saccade-related activity in its deeper layers. Since collicular movement fields are quite large, a considerable region of the colliculus is active whenever a saccade is made. We have modelled the collicular role in saccade generation based on the idea, proposed earlier in the literature, that each movement cell causes a movement tendency in the direction of the external world point which it represents in the collicular map. The model is organized as follows: (1) An anisotropic logarithmic mapping transforms retinal coordinates into collicular coordinates. (2) A two-dimensional Gaussian function describes the spatial extent of the movement-related activity in the deeper layers. (3) An efferent mapping function specifies how the direction and the size of the movement contribution of each colliculus neuron depends on its location and its firing rate. (4) The total saccade is the vector sum of the individual cell contributions.

This very simple model (seven fixed parameters) has been used to simulate metrical properties of saccades: (a) in response to visual targets; (b) in response to electrical stimulation in one colliculus, and (c) after a colliculus lesion. Model performance appears to be remarkably realistic but cannot account for some border effects and responses to double stimulation. Suggestions on how the model can be improved and extended will be presented.
\end{abstract}

In this paper we will present a quantitative model which attempts to describe the relation between neural activity in the superior colliculus (SC) of the monkey and the metrics of the resulting saccade. The model extends earlier work by Ottes et $_{\text {al }}{ }^{23}$ and relies heavily on ideas expressed by McIlwain ${ }^{19.20}$ and Sparks et al ${ }^{38}$ One of its main purposes is to explore, at the quantitative level, the implications of the well-established fact that the generation of a saccade always involves a population of recruited SC neurons. Since any efferent SC neuron will participate in a range of saccade sizes and directions, it is far from trivial to predict off-hand the full implications of schemes based on fixed spatially weighted connection strengths with brainstem burst neurons. ${ }^{9} 16,39$ Before the model is discussed we will review the relevant literature. For more extensive reviews we refer elsewhere. ${ }^{9,35,39,45}$

The SC is a layered structure which contains a neural map of the retina in its superficial layers and spatially coded motor-related activity, occurring before and during saccades, in its deeper layers. The most direct way to study the topographical organization of the visual activity is to plot visual receptive fields of neurons throughout the SC. It is known that the position of the visual receptive field on the retina depends systematically on the anatomical location of the neuron within the SC, but the

*Author to whom correspondence should be addressed. Abbreviations: SC, superior colliculus; MLB, medium-lead burst cell; LLB, long-lead burst cell. data available are too incomplete to serve as a basis for our model. Similarly, studies on SC movement cells in the deeper layers have disclosed that the saccade vector associated with the most vigorous discharge in a particular cell (the optimal saccade) is related to its anatomical position within the SC. Again, these data are too fragmentary to be used for a mathematical description of the SC maps which is. an important element in our model. It is possible, however, to reconstruct the topographical organization of the SC indirectly from electrical stimulation data. ${ }^{29}$ Schiller and co-workers ${ }^{32,33}$ have shown that the saccade vector elicited by local SC stimulation is roughly identical with the location of the visual receptive field and the optimal saccade vector in the movement field of nearby neurons. By relying on this finding (Schiller's relationship, for short), the saccade vectors evoked by electrical stimulation at many different points in the $\mathrm{SC}^{29}$ can be used to reconstruct, indirectly, the structure of the afferent mapping. Using this approach, Ottes et al. ${ }^{23}$ have given a mathematical description of the topographical organization of the afferent mapping. Their description allows one to specify where in the SC visual map a target at a certain retinal location will cause maximal neural activity.

For the purpose of our model the location of the movement-related activity in the deeper layers is of special importance. Based on the relationships found by Schiller's group, ${ }^{32,33}$ it is reasonable to assume that the movement-related activity is maximal at the same location where the target caused maximal visual 

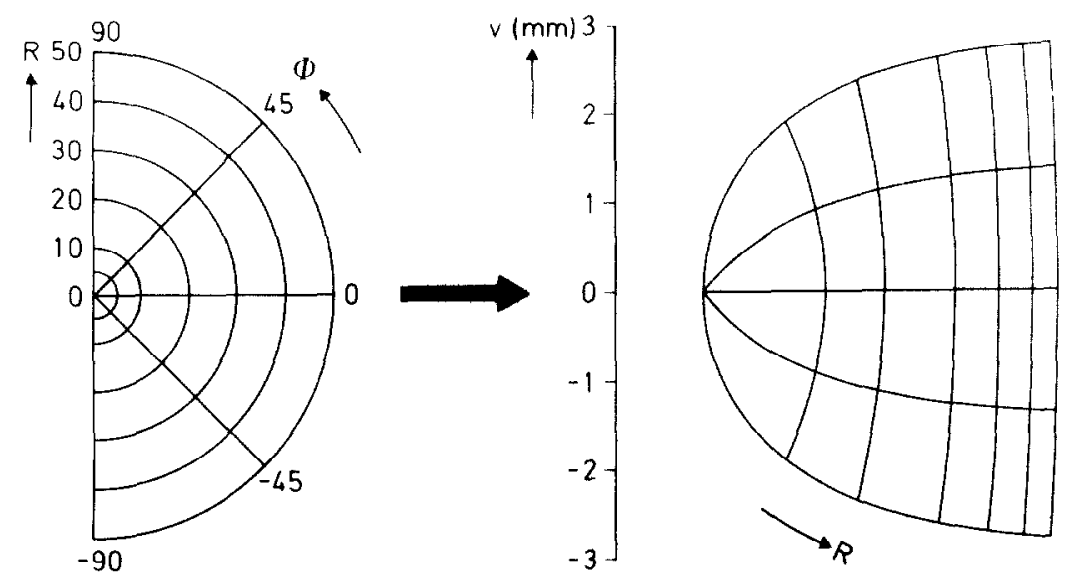

A
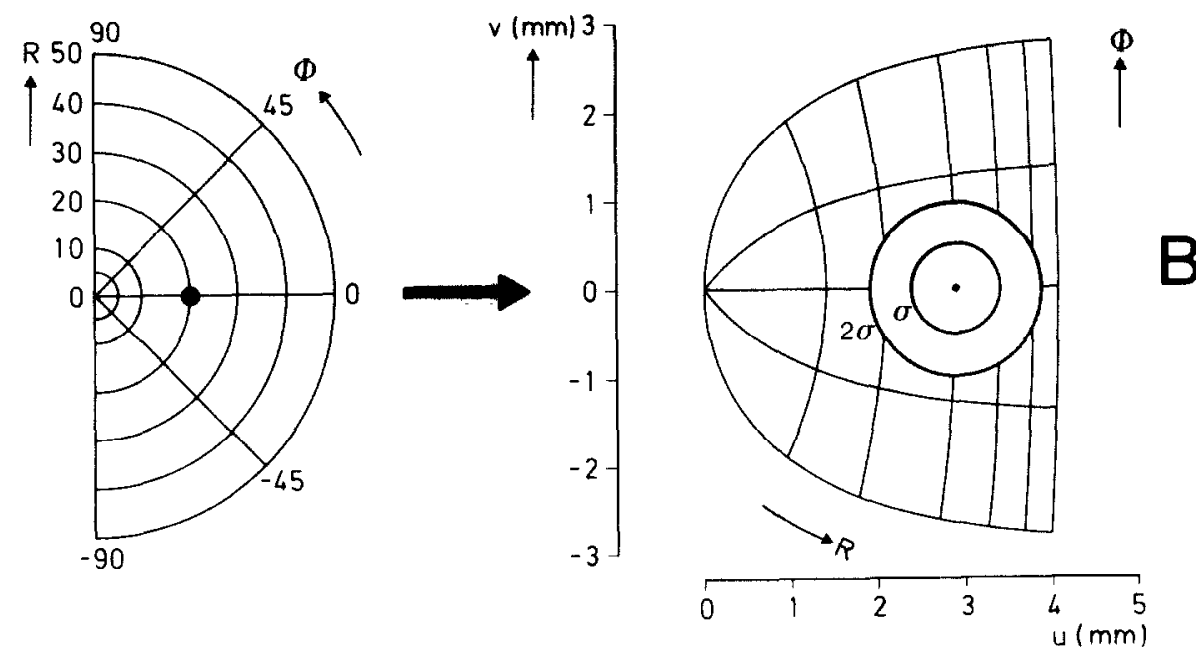

Fig. 1. Spatial distribution of movement-related activity in SC. (A) The afferent mapping lequations (1) and (2)] determines the relation between retinal target location (left-hand side) and the locus of maximum SC movement-cell activity $\left[\left(u_{c}, v_{c}\right)\right.$; right-hand side]. The polar coordinate grid on the retina has meridians every $45 \mathrm{deg}$ and isoeccentricity hemicircles of $5,10,20,30,40$ and $50 \mathrm{deg}$. Largest eccentricity represented in SC in the model equals 100 deg (not shown). Mapping parameters as in text. (B) Spatial distribution of movement-related activity in SC when target is presented at $R=20 \mathrm{deg}$ and $\Phi=0 \mathrm{deg}$. Heavy dot in SC map denotes $\left(u_{c}, v_{c}\right)$ the locus of maximum movement-cell activity, specified by the afferent mapping (A). Circles $\sigma$ and $2 \sigma$ denote width of Gaussian weighting function [equation (3)].

activity in the superficial layers. Thus, the same point-to-point mapping which relates stimulus position and the location of visual peak activity can also serve to describe the locus of the most vigorous saccade-related activity. Since it is known that the visual receptive field and the movement field of SC visuomotor neurons are nested, ${ }^{23,46}$ this is an entirely reasonable assumption. It ties in with the generally held belief that the subjacent representations of visual and motor activity in the SC are isomorphic and in register.

It would be very unsatisfactory, in modelling SC function, to consider only the afferent mapping (Fig. 1A) and to ignore the obvious fact that a target stimulus, which elicits a saccade, creates activity, not just at one point, but in an entire region of the visual and the motor map (Fig. 1B). For our purpose, the spatial extent of the motor activity in the deeper layers is of special importance. Whether this activity originates in the upper layers or elsewhere is irrelevant in the present context as long as its spatial location fits in with our description of the afferent mapping (see below). How can an estimate of the spatial distribution of the movement-related activity (the neural population activity profile) be obtained?

To reconstruct the spatial distribution of movement-related activity associated with saccades, the size of collicular movement fields is of obvious 
importance. To obtain the movement field of a single neuron one usually measures mean firing rate in the burst $(F)$ as a function of saccade amplitude $(R)$ and direction $(\Phi)$. From $F(R, \Phi)$ plots, which then can be constructed, it is known that a given neuron may be active in a wide amplitude/direction range of saccades. ${ }^{23,38,46}$ Because of this fact, which implies that SC movement cells are also active for nonoptimal saccades with somewhat deviating amplitudes and directions, a cluster of cells will always be recruited for any given saccade. Assuming that cell density in the collicular map is constant and that neurons with various optimum saccade sizes are equally numerous (homogeneous mapping), the number of neurons recruited for each saccade is fully determined by the distribution of movement field sizes. Since neurons with large optimum saccade vectors have larger movement fields, ${ }^{38,46}$ in this hypothetical case relatively more $\mathrm{SC}$ movement cells would be involved in the generation of large saccades. However, the SC mapping is actually nonhomogeneous: movement cells with small optimum saccade vectors occupy a relatively large amount of SC map space. ${ }^{23}$ Therefore, both the size of the movement field and the properties of the mapping must be taken into account if one wishes to reconstruct the population activity profile from single cell data.

A procedure which leads to the desired result is to replot movement fields, from external world coordinates expressed in degrees $[F(R, \Phi)]$, into collicular coordinates expressed in millimeters $[F(u, v)]$. Here $(u, v)$ are collicular coordinates assigned to $(R, \Phi)$ by the afferent mapping. When such a converted plot is made for the data of a single SC movement cell, ${ }^{23}$ one obtains a roughly rotationally symmetrical domeshaped function which has its maximal value (peak firing rate) at its collicular location which corresponds with its own particular optimal saccade vector. A saccade with a somewhat different amplitude and/or direction will cause maximal activity in cells located at a corresponding nearby SC location, but will be associated with a lower firing rate in the cell whose data are plotted. How rapidly the cell's firing rate falls off, when the location of maximal excitation shifts in the map as the saccade deviates more and more from its own optimal saccade vector, can directly be seen in such a converted movement-field plot. By extrapolating from such single cell data, we have been led to assume in the model that the population activity profile of SC movement cells also has a dome-shaped profile. Since, so far, there are no data to indicate otherwise, we have assumed that the population activity profile is translation-invariant so that only its location, unlike its size and shape. depends on target location.

The spatial extent of the dome-shaped function, reconstructed in a small sample of monkey SC neurons ${ }^{23}$ has a diameter in the order of millimeters. This is quite large compared with the total size of the $\mathrm{SC}$ in the monkey (see below). This measure will be used as the best estimate yet available for the spatial distribution of SC movement-cell activity.

The circumstance that a population of active cells in the SC motor map, whose location shifts with target location, and activates the saccade generator circuits downstream such as to generate a normometric saccade, is a formidable challenge for any quantitative model of collicular function. This problem has led McIlwain ${ }^{19,20}$ and Sparks et al ${ }^{38}$ to make suggestions on how SC population activity may create the appropriate movement. They proposed that each SC movement cell has functional connections with the horizontal and vertical movement generating systems in the reticular formation, such that its activity creates a movement tendency in the direction of the external world point which it represents in the SC map. The total saccade, then, would be the total vector sum of all these movement contributions. This proposal for ensemble coding of saccades in the SC has not been investigated quantitatively, so far. In this paper we have put these ideas in the form of a specific and very simple model of SC saccade coding. With the model we endeavoured to test whether ensemble coding, inherent in these ideas, could work in principle. Simulations made with the model show how it can be tested against experimental data obtained in electrophysiological, electrical stimulation and lesion experiments.

\section{THE MODEL}

Our description of the model is subdivided as follows: (1) a description of the afferent mapping in the monkey SC; (2) a description of the population activity profile of movement-related cells; and (3) a description of how the spatial distribution of recruited movement cells creates movement.

\section{The afferent mapping}

In the present paper we will use the results obtained by Ottes et $a .^{23}$ as a model of the afferent mapping in the monkey SC. In the Introduction we have explained how electrical stimulation data can be used, indirectly, to characterize the afferent mapping. In fitting Robinson's data, ${ }^{29}$ the best results were obtained with an anisotropic logarithmic mapping model:

$$
\begin{aligned}
& u=B_{u} \ln \left[\frac{\sqrt{R^{2}+A^{2}+2 A R \cos \Phi}}{A}\right] \\
& v=B_{v} \operatorname{atan}\left[\frac{R \sin \Phi}{R \cos (\Phi)+A}\right]
\end{aligned}
$$

where:

$u=$ anatomical distance from the foveal representation in the SC measured along the horizontal meridian representation $(\mathrm{mm})$

$v=$ anatomical distance along the $v$-axis which is perpendicular to the $u$-axis (mm) 
Table 1. Parameter values used in simulations

\begin{tabular}{ll}
\hline Parameter & \multicolumn{1}{c}{ Value } \\
\hline$B_{u}$ & $1.4 \mathrm{~mm}$ \\
$B_{t}$ & $1.8 \mathrm{~mm} / \mathrm{rad}$ \\
$A$ & $3.0 \mathrm{deg}$ \\
$F_{\max }$ & $500 \mathrm{spikes} / \mathrm{s}$ \\
$\sigma$ & $0.5 \mathrm{~mm}$ \\
$\alpha$ & $3.57 \times 10^{-\sigma}(\text { spikes } / \mathrm{s})^{-1}$ \\
$R_{\lim }$ & $100 \mathrm{deg}$ \\
\hline
\end{tabular}

$B_{u}=$ a scaling constant determining the size of the SC map along its $u$-axis (mm)

$B_{v}=$ a scaling constant determining the size of the SC map along its $v$-axis ( $\mathrm{mm} / \mathrm{rad})$

$A=$ a constant which, together with the ratio $B_{u} / B_{v}$, determines the shape of the mapping (deg)

$R=$ retinal eccentricity of target (deg)

$\Phi=$ meridional direction of target (deg).

This model is equivalent to the complex logarithmic mapping model used by Schwartz ${ }^{36}$ in monkey area 17 if $B_{u}=B_{v}$. In the present paper the parameter values will remain fixed at their optimal values for the $\mathrm{SC}$ in the monkey: ${ }^{23} \quad B_{u}=1.4 \mathrm{~mm}$; $B_{v}=1.8 \mathrm{~mm} / \mathrm{rad}$ and $A=3.0 \mathrm{deg}$.

With equations (1) and (2) it is possible to describe the representation of a hemiretina on the contralateral colliculus (Fig. 1A). We assume that the collicular map does not extend beyond 100 deg eccentricity ( $R_{\text {lim }}$; see Table 1$)$. In the simulations, equations (1) and (2) were used to compute which movement cell is maximally excited by a given target location.

\section{The population activity profile in the motor map}

In the model we approximate the dome-shaped population activity profile inferred from $\mathrm{SC}$ movement cell data with a two-dimensional Gaussian weighting function: $:^{23}$

$$
\begin{gathered}
F=F_{\max } \cdot \exp \left[-\frac{\left(u-u_{c}\right)^{2}+\left(v-v_{c}\right)^{2}}{2 \sigma^{2}}\right] \\
F=0 \text { outside the } \mathrm{SC} \text { and beyond } \\
2 \sigma \text { from }\left(u_{c}, v_{c}\right)
\end{gathered}
$$

where:

$F=$ mean firing rate in the burst of movement cell at position $(u, v)$ in the SC (spikes/s)

$F_{\text {max }}=$ maximum mean burst rate in the population (spikes/s)

$\left(u_{c}, v_{c}\right)=$ location of most active movement cell in the population ( $\mathrm{mm}$ ) which is determined by target location and the mapping equations (see above)

$\sigma=$ parameter determining decay rate of neural activity in the population with distance from $\left(u_{c}, v_{c}\right)$ in the map $(\mathrm{mm})$.

The Gaussian weighting function extends indefinitely. This property would imply that a target anywhere on the retina would create at least some activity throughout the SC. Since this may be unrealistic, the outer rim of the Gaussian weighting function was truncated beyond a distance of $2 \sigma$ from $\left(u_{c}, v_{c}\right)$. Of course, when Gaussian weighting function transgressed the border of the SC, it was also truncated [see equation (3)]. The border of the colliculus $\left(R_{\text {lim }}\right)$ was taken at the $R=100 \mathrm{deg}$ representation.

In the simulations we used fixed values for the parameter $F_{\max }(500$ spikes $/ \mathrm{s})$ and $\sigma(0.5 \mathrm{~mm})$. The $\sigma$ value of $0.5 \mathrm{~mm}$ corresponds roughly to the mean value computed from a small sample of monkey $\mathrm{SC}$ movement cells recorded by Ottes et al. ${ }^{23}$ The effects of changing $\sigma$ will be considered later (see Results).

The set of equations (1), (2) and (3) gives a complete description of the location and the spatial extent of the movement-related activity for saccades to a target anywhere on the retina (Fig. 1A, B) except for one special case. The fact that the collicular representation is split into two separate maps creates a complication if one considers how the movementrelated activity in our model would look like when the visual target is on the vertical meridian. How is the movement-related activity distributed in this case? The most logical extension of the line of reasoning so far, in line with the generally accepted idea that purely vertical saccades require signals from both colliculi, ${ }^{29}$ is to assume that in this case the population activity profile is split up into two equally sized parts which have their peak value on the vertical meridian representation in each colliculus at the locations which correspond with the target's retinal eccentricity. If the target is presented on a meridian just off the vertical, so that now the saccade requires also a small horizontal component, the dome should be split into two unequal parts with most of the activity in the contralateral colliculus. The visual representation in the superficial layers of the monkey SC represents only the contralateral half field ${ }^{5}$ but there is evidence that (nearly) vertical saccades, indeed, recruit SC movement cells in both colliculi. ${ }^{38,46}$ To make simulations with the model when this kind of border effect occurs, specific assumptions had to be made about the movement-related activity in each colliculus. The procedure used to compute the spatial distribution of movement-related activity in each colliculus, when the visual target was near the vertical meridian, is explained in the Appendix.

In simulations of electrical stimulation with a single electrode in one motor colliculus (see below) we assumed that the electrical stimulus creates the activity described by equation (3). In this case $\left(u_{r}, v_{c}\right)$ corresponds with the electrode tip position in the collicular motor map and the activity profile remains confined to the stimulated colliculus.

\section{From superior colliculus neural activity to movement}

To explain how the model transforms movementcell activity into movement we assign a vector, $\mathbf{P}$, to each $\mathrm{SC}$ neuron. This vector represents the potential 

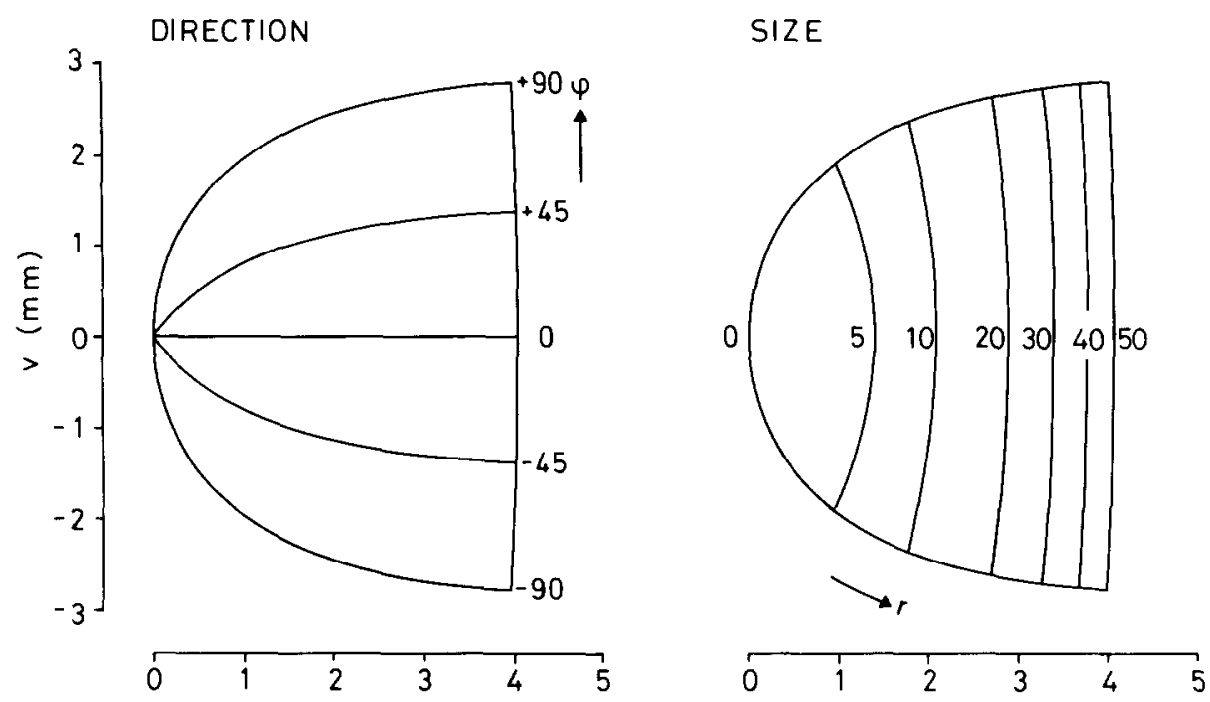

Fig. 2. Movement potential vector field in SC. Lines in the SC map on the left (cf. Fig. 1A) connect cells which generate movement contributions in the same direction [equation (4)]. Lines in the SC map on the right indicate relative size of the movement potential vector as a function of SC location [equation (5)]. Numbers in arbitrary units.

of each cell to create movement once it is recruited into activity. The direction of the movement potential vector $\mathbf{P}_{\mathrm{i}}$ of a given cell $i$ is determined by the relative strengths of its connections with the horizontal and the vertical movement systems downstream. Its size is determincd by the absolute strengths of these connections (see Discussion). We propose that size $(r)$ and direction $(\phi)$ of the movement potential vector depend systematically on the $(u, v)$ position of the neuron in the SC map according to an inverse mapping relationship ${ }^{23}$ (see Fig. 2):

$$
\begin{aligned}
& r=A \sqrt{\exp \left(\frac{2 u}{B_{u}}\right)-2 \exp \left(\frac{u}{B_{u}}\right) \cdot \cos \left(\frac{v}{B_{v}}\right)+1} \\
& \phi=\operatorname{atan}\left[\frac{\exp \left(\frac{u}{B_{u}}\right) \cdot \sin \left(\frac{v}{B_{v}}\right)}{\exp \left(\frac{u}{B_{u}}\right) \cdot \cos \left(\frac{v}{B_{v}}\right)-1}\right]
\end{aligned}
$$

with, again, $B_{u}=1.4 \mathrm{~mm} ; B_{v}=1.8 \mathrm{~mm} / \mathrm{rad}$ and $A=3 \mathrm{deg}$. For the explanation of symbols we refer to equations (1) and (2).

In the model we assume that the movement contribution, $\mathbf{M}_{i}$, of a given movement cell $i$ depends only on its movement potential vector, which is fixed, and the mean burst rate:

$$
\mathbf{M}_{i}=\alpha \cdot F_{i} \cdot \mathbf{P}_{i}
$$

where

$\mathbf{M}_{i}=$ the movement vector contribution of cell $i$

$\alpha=$ a fixed scaling constant (spikes/s) ${ }^{-1}$

$F_{1}=$ the mean burst rate of cell $i$ (spikes/s) which is fully determined by target location and equations (1), (2) and (3)

$$
\begin{aligned}
& \mathbf{P}_{i}= \text { the movement potential vector of cell } i \text { [see } \\
& \text { equations (4) and (5)]. }
\end{aligned}
$$

We emphasize that $\alpha$ is the same for all cells and is independent of firing rate.

Equation (6) is the simplest expression we could think of to formulate the ensemble coding hypothesis, ${ }^{19,20,38}$ in mathematical terms. What does equation (6) mean? Since the movement potential vector of each cell is related to its anatomical location, and therefore fixed, the direction of its movement contribution is likewise predetermined; only its amplitude can vary. According to equation (5) the direction of the movement contribution of any cell corresponds with the retinal meridian which it represents in the SC map. Accordingly, all active cells on the horizontal meridian representation will generate a purely horizontal movement contribution. Cells on the vertical meridian representation will contribute a vertical movement vector, etc. Since a target causes movement cell activity in an entire region of the SC map, the movement contributions of many cells will have somewhat wrong directions. This does not mean that the scheme cannot work because movement contributions which are wrong in one direction are compensated by others which are off in the mirror direction (Fig. 3).

The amplitude of the movement contribution of a given cell depends on the size $(r)$ of its movement potential vector and its firing rate [equation (6)]. If the cell is silent it does not contribute at all. When it is recruited the size of its movement contribution is proportional to the cell's firing rate, no matter how this firing rate came about. For simplicity, the assumption that the effect is proportional to mean firing 

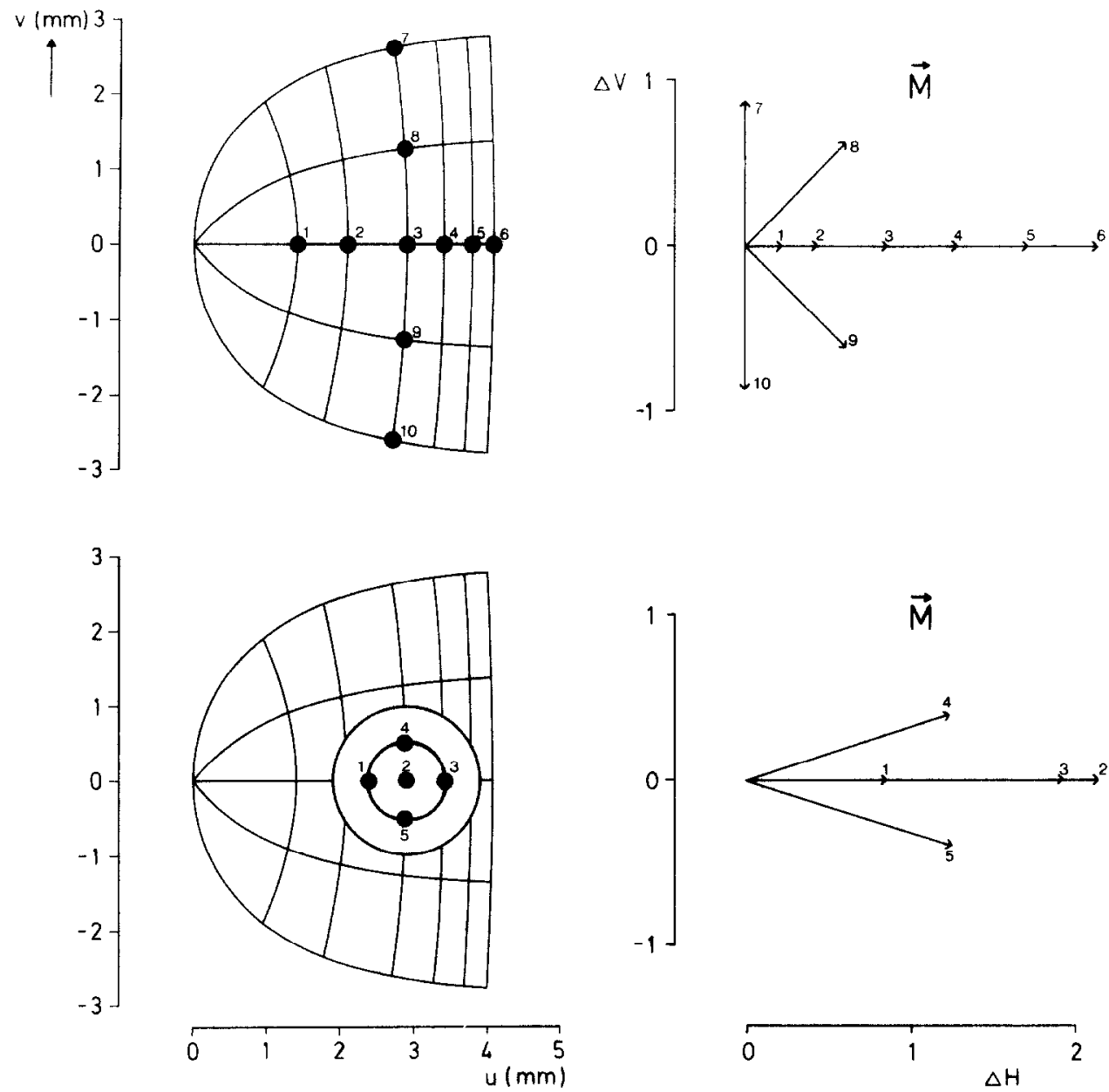

Fig. 3. Efferent mapping in the model. (A) Dependence of movement contribution vector $\mathbf{M}$ (right-hand side) on location of SC neuron (left-hand side). For a given fixed firing rate, 200 spikes/s in this example, neurons 1 through 10 have different $\mathbf{M}$ vectors which reflect their different movement potential (see Fig. 2). (B) Combined effect of firing rate and SC location on movement contribution $\mathbf{M}$. The figure on the left shows the Gaussian population activity profile for a target at $R=20 \mathrm{deg}$ and $\Phi=0 \mathrm{deg}$ (see Fig. IB). The five identified neurons have firing rates of 500 spikes/s (neuron 2) and $500 \times e^{-0.5}=303$ spikes/s (neurons 1, 3, 4 and 5). Cells 1, 2 and 3 contribute purely horizontal $\mathbf{M}$ vectors whose sizes are proportional to the product of each neuron's firing rate and the size of its movement potential vector [see Fig. 2 and equation (6)]. Note that cells 4 and 5 contribute partially misdirected $M$ vectors. Scale in drawings on right-hand side in minutes of arc.

rate in the burst disregards possible postsynaptic nonlinearities (see Discussion). It follows from equation (4) that the size of the movement potential vector $\mathbf{P}_{i}$ increases dramatically (roughly exponentially) as one moves in the SC map from a position near the foveal representation away to the border of the colliculus. These and other propertics of the model are illustrated graphically in Fig. 3.

The total effect of the activity in the population of SC movement cells in the model is obtained simply by vector summation of the movement contribution of the individual cells:

$$
\mathbf{S}=\sum_{i=1}^{N} \mathbf{M}_{i}
$$

where $\mathbf{S}$ is the resulting saccade vector and $N$ is the total number of cells in the SC motor map.
It is often assumed that the SC determines only the metrics of the desired saccade and that the dynamic properties of the movement are determined downstream of the colliculus. If we adhere to this view, $S$ in equation (7) would be the desired saccade vector. The model is linear in the sense that the effect of the total population of cells is a simple addition of individual contributions. It seemed a sensible approach to us to explore what a linear summation model can explain before studying the effect of nonlinear interactions.

\section{EXPERIMENTAL PROCEDURES}

\section{Computer simulations}

To make simulations with the model, we considered the motor colliculus as an equal-density two-dimensional square lattice of neurons arranged in rows and columns 


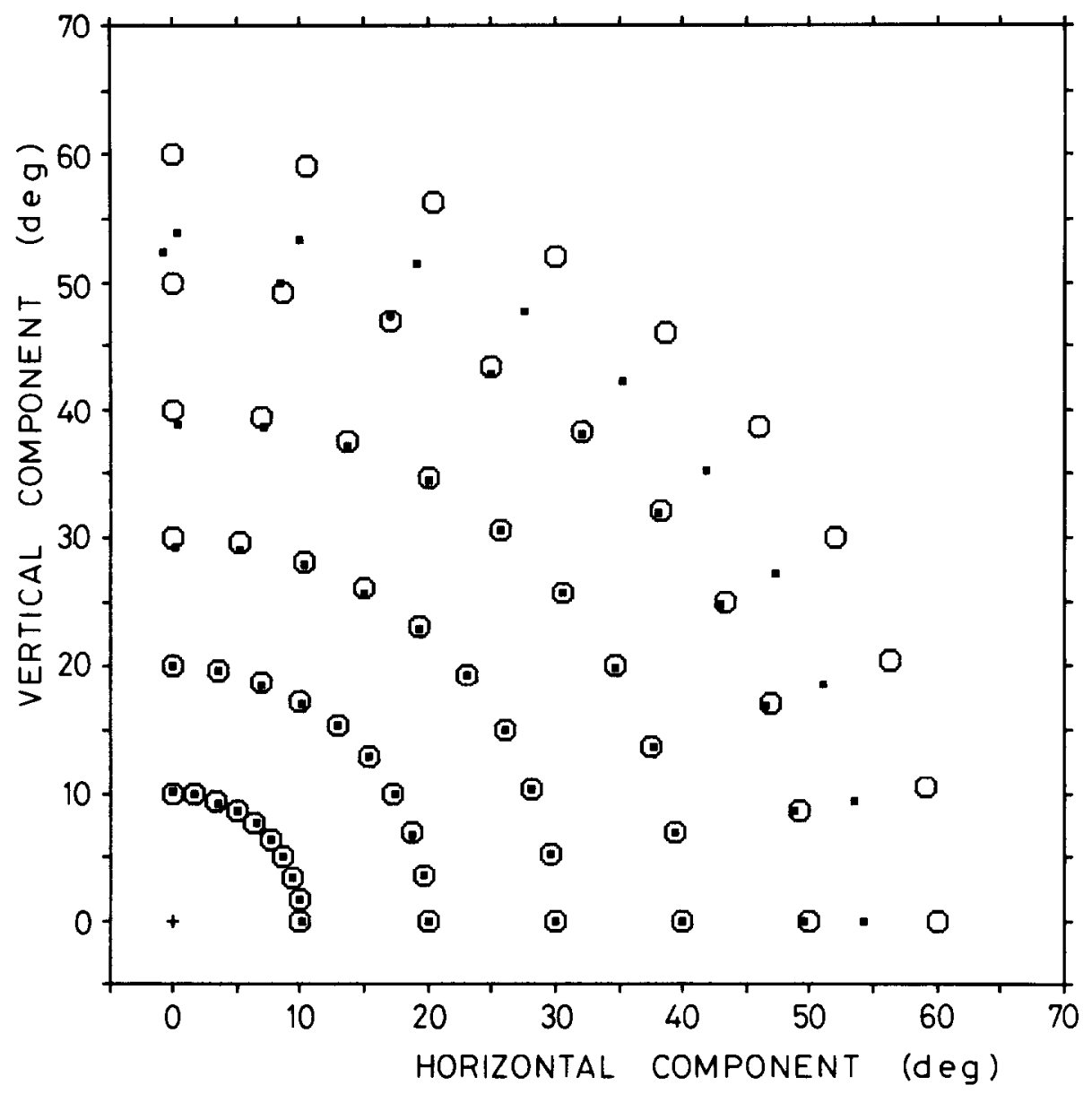

Fig. 4. Model responses to visual targets in first quadrant. Responses (dots) are close to the target (open circles), except in the far periphery where amplitudes fall short.

along the $u$ - and the $v$-axis, respectively. The distance between neighbouring cells was arbitrarily taken as $50 \mu \mathrm{m}$. If cell density would be made larger, this would merely lead to a smaller $\alpha$ value in the model. The borders of each colliculus were taken as the vertical meridian and the $R=100$ deg representation.

The constant $\alpha$ in equation (6) was determined as follows. The target was placed at $R=20 \mathrm{deg}$ on the horizontal meridian $(\Phi=0 \mathrm{deg})$. In this case the entire population of active cells is contained completely in one colliculus without infringing on its borders. We found that the model yielded a normometric saccade of very good precision if $\alpha$ had a value of $3.57 \times 10^{-6}$ (spikes/s) $)^{-1}$. In all further simulations, $\alpha$ was kept fixed at this value. Given the assumptions made, the contribution of one cell to the total saccade, even if it is firing at 500 spikes/s, is very small. For example, when the target is at $R=20 \mathrm{deg}$ and $\Phi=0 \mathrm{deg}$ the maximally excited SC cell (neuron 2 in Fig. 3B) will contribute a horizontal vector of:

$$
\begin{aligned}
\alpha \cdot F \cdot \mathbf{P} & =3.57 \times 10^{-6} \times 500 \times 20 \\
& =0.0357 \mathrm{deg}(2.14 \mathrm{~min} \text { of arc }) .
\end{aligned}
$$

\section{List of parameters}

For convenience, we now list the parameters as they were used in the computer simulations unless noted otherwise (Table 1).

\section{RESULTS}

\section{Model responses to visual target presentation}

Using the fixed $\alpha$-value found by tuning the model to make a correct response to a target at $(R, \Phi)=(20,0)$ (see Experimental Procedures), the model's response $\mathbf{S}$ was computed for a wide range of target locations in the first quadrant. This is obviously an essential test to find out whether the model, despite its extreme simplicity (a fixed $\alpha$ value equal for all cells), can really generate normometric saccades. The results of the computer simulations are shown in Fig. 4.

It is clear from the generally excellent correspondence between target location and the metrics of the model response that the model stands this test remarkably well (see, however, below).

As we made clear already above, a complicating factor in the analysis of spatial saccade coding is the occurrence of border effects. In the case of a visual stimulus near the vertical meridian, there are two populations of active movement cells, one in each colliculus. Their precise location and size depend on the exact target location and, in the simulations, 

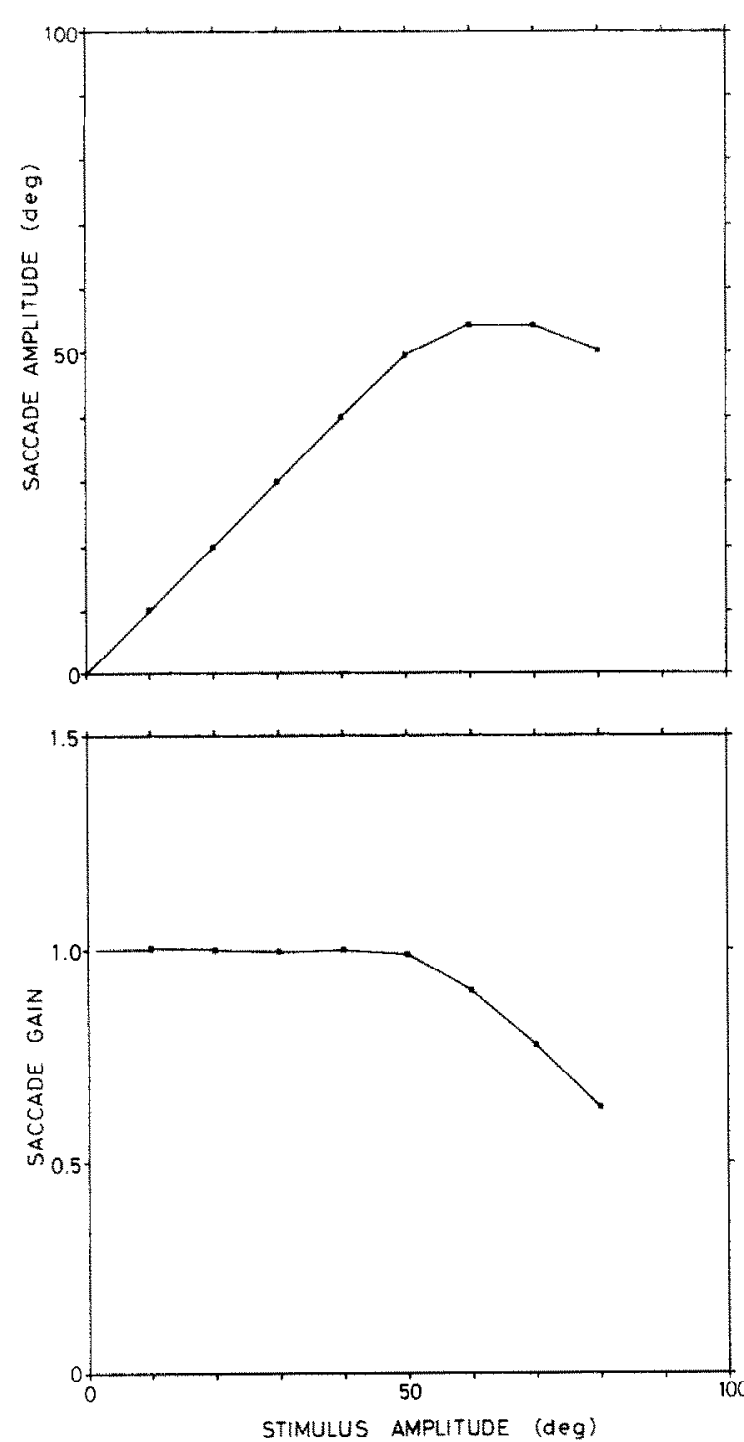

Fig. 5. Response amplitude as a function of target eccentricity. Upper panel: as stimulus eccentricity increases, saccade size increases to a saturation level of about $55 \mathrm{deg}$ and then falls back slightly due to border truncation of movement cell activity. Lower panel: saccade gain decreases from 1.0 to lower levels for targets beyond $50 \mathrm{deg}$.

followed rules outlined in the Appendix. Although these rules were only meant to be pragmatic approximations, the simulation results are still quite satisfactory for target presentations near the vertical meridian.

Border effects can also occur near the model's $R=100 \mathrm{deg}$ eccentricity limit $\left(R_{\mathrm{lim}}\right.$ in Table 1$)$. The simulations show that, with the present parameter values, the model cannot make saccades larger than about $55 \mathrm{deg}$. The reason behind this is that, as the active population of movement cells shifts to the eccentricity limit in the colliculus, the number of movement contributing cells drops more and more because of border truncation [see equation (3)]. In the extreme, when target eccentricity is very large, the target will not be "seen" by the SC and evoke no saccade at all. Thus, as target eccentricity increases, the gain in the model is approximately unity up to $R=50 \mathrm{deg}$, and then drops off gradually to zero. As a consequence, its response to an enlarged stimulus displacement may be a saccade which is actually smaller. These effects are illustrated in Fig. 5 and will be further discussed below (Discussion).

\section{Model simulations of electrical stimulation experi- ments}

In simulations of Robinson's electrical stimulation data,,$^{29}$ the neural population activity was limited to one colliculus. The results are quite satisfactory for most data points (Fig. 6). The stimulation points where the discrepancies are largest are those where the mapping model gives a rather poor fit. ${ }^{23}$ Thus, the slight imperfections of the anisotropic logarithmic mapping model may explain why the fit between experimental data and model result was good but not perfect.

In other simulations we found that the model obeys Schiller's relationship (see Introduction) except when the electrical stimulation site is so close to $R_{\text {lim }}$ (see Table 1) or the vertical meridian border of the colliculus that border truncation effects arise. A typical result is shown in Fig. 7 where electrical stimulation was simulated for various sites on the $R=30 \mathrm{deg}$ representation in the left SC. The model saccade vector, shown by arrows in Fig. 7, fits Schiller's relationship as long as the activity profle in the colliculus does not reach the vertical meridian border. When the electrode is simulated to be on the vertical meridian border, there is a severe mismatch between the locally represented $R$ and $\Phi$ coordinates and the evoked saccade vector. In these cases, the saccade is much too small and its direction is off by some $12 \mathrm{deg}$. The latter result fits in nicely with the remark in Robinson's paper that saccades with directions deviating less than 10-20 deg from pure vertical typically could not be elicited with a single electrode (see Discussion).

The border effects at the $R=100 \mathrm{deg}$ border, as expected, were entirely similar to the undershoots to visual targets at very large eccentricity (see Figs 4 and 5).

\section{Simulation of lesion effects}

Simulations were made to predict the effects produced by inactivating all cells in a $1 \mathrm{~mm}$ diameter circular area around the point in the SC map representing $R=20 \mathrm{deg}$ and $\Phi=0 \mathrm{deg}$. The lesion was simulated by assuming that the affected cells were silent $(F=0)$ under all stimulus conditions. Of course, when the normal population activity profile (Fig. 1B) overlaps with the inactivated region, the movement contribution of some cells will be missed and this will cause the saccade to become hypometric. The saccade will also be slightly off-axis (directed away from the lesion) for target presentations which 

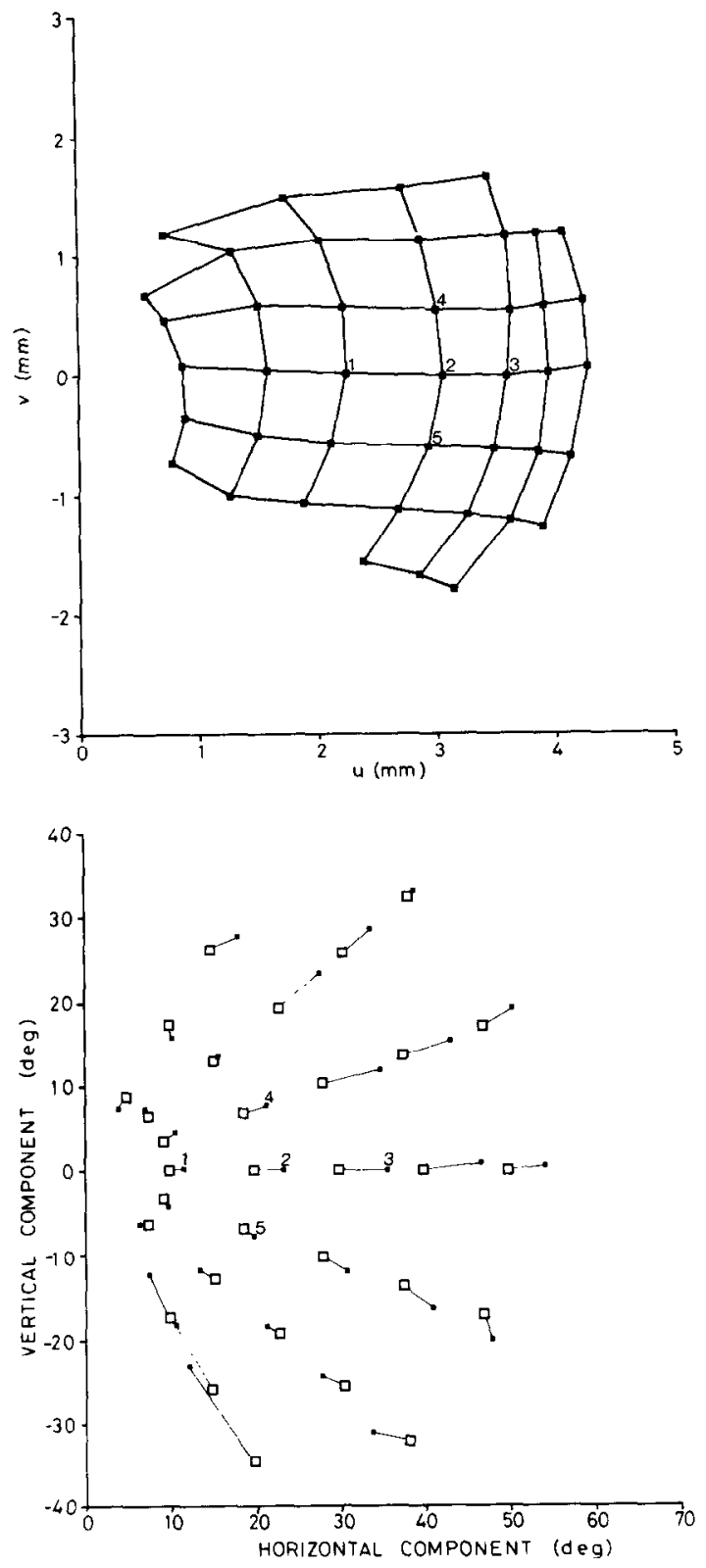

Fig. 6. Simulation of response to electrical stimulation in one colliculus. Upper part: set of $(u, v)$ points in SC map where the result of electrical stimulation is known. Sec Fig. 4B in Robinson's paper. ${ }^{29}$ Lower part: comparison of experimental and model saccade responses for stimulation sites shown in upper panel. Open squares: experimental. Dots: model. The model results were obtained using the fixed set of parameters (see text) which was also used to obtain the data in Figs 4, 5, 7 and 8. Experimental and theoretical data for 2 and $5 \mathrm{deg}$ saccades were in very good agreement but are not shown here. Correspondence between points in both panels is specified by a few numbered examples.

are not on the horizontal meridian. In the computer simulations target stimuli were presented on 5 meridians and 10 eccentricities (Fig. 8). The results show the saccadic undershoot quite clearly; the direction offset is a more subtle phenomenon. As can be seen,
20 deg horizontal saccades can still be generated after the lesion, but only in response to target presentation at a larger eccentricity. Much stronger effects were obtained with a $2 \mathrm{~mm}$ diameter lesion (not shown). These effects will be compared with literature data in the Discussion.

Shape of superior colliculus movement fields. We have also simulated the shape of SC movement fields (not shown). We found that movement fields in the model, as in the monkey, ${ }^{23,38}$ are skewed along the $R$-dimension and symmetrical along the $\Phi$-dimension (see Introduction). However, large eccentricity model neurons $(R>50 \mathrm{deg})$ did not have a unique $F(R)$ relation. This phenomenon and the rather too small oculomotor range may well reflect a common shortcoming in the model (see Discussion).

Effect of changing parameter $\sigma$. Simulations were also made for $\sigma$ values of 0.75 and $1.0 \mathrm{~mm}$. After adjustment of the $\alpha$ parameter, the model again generated normometric saccades but border truncation effects became more severe so that the oculomotor range became even smaller than in Figs 4 and 5. The violation of Schiller's relationship in the simulation results became stronger than in Fig. 7 and seemed exaggerated compared to Robinson's observations. ${ }^{29}$ Based on these considerations, a $\sigma$-value exceeding $0.5 \mathrm{~mm}$ (Table 1) was not considered realistic.

\section{DISCUSSION}

\section{Evaluation of the vector-summation model}

The performance of the vector-summation model, considering its extreme simplicity, is quite remarkable. The results obtained show that SC ensemble coding of saccades is a very promising hypothesis. The idea of ensemble coding in movement control, first proposed for the saccadic system, ${ }^{19,20,38}$ has recently also been applied to the control of arm movements. ${ }^{10.11}$

Our model tries to account for the relation between the spatial pattern of activity in SC movement cells and the metrical properties of saccadic eye movements. Since it is generally assumed that the dynamical properties of saccades reflect the operation of neural circuits downstream of the SC, we have only considered the spatial properties of saccades. The simulation studies have yielded interesting results which, when judged qualitatively, look quite realistic. The model has its shortcomings, of course, and these will come to light as we discuss the various simulation results in more detail.

Saccades to visual targets. These simulations yielded normometric saccades for targets up to about 50 deg eccentricity in all directions. The limited extent of the afferent map in our model, which leads to border truncation of the population activity profile, causes the oculomotor range to be limited as well. If, as is generally assumed, the limited oculomotor range of + and $-45 \mathrm{deg}$ in man and monkey is due to 

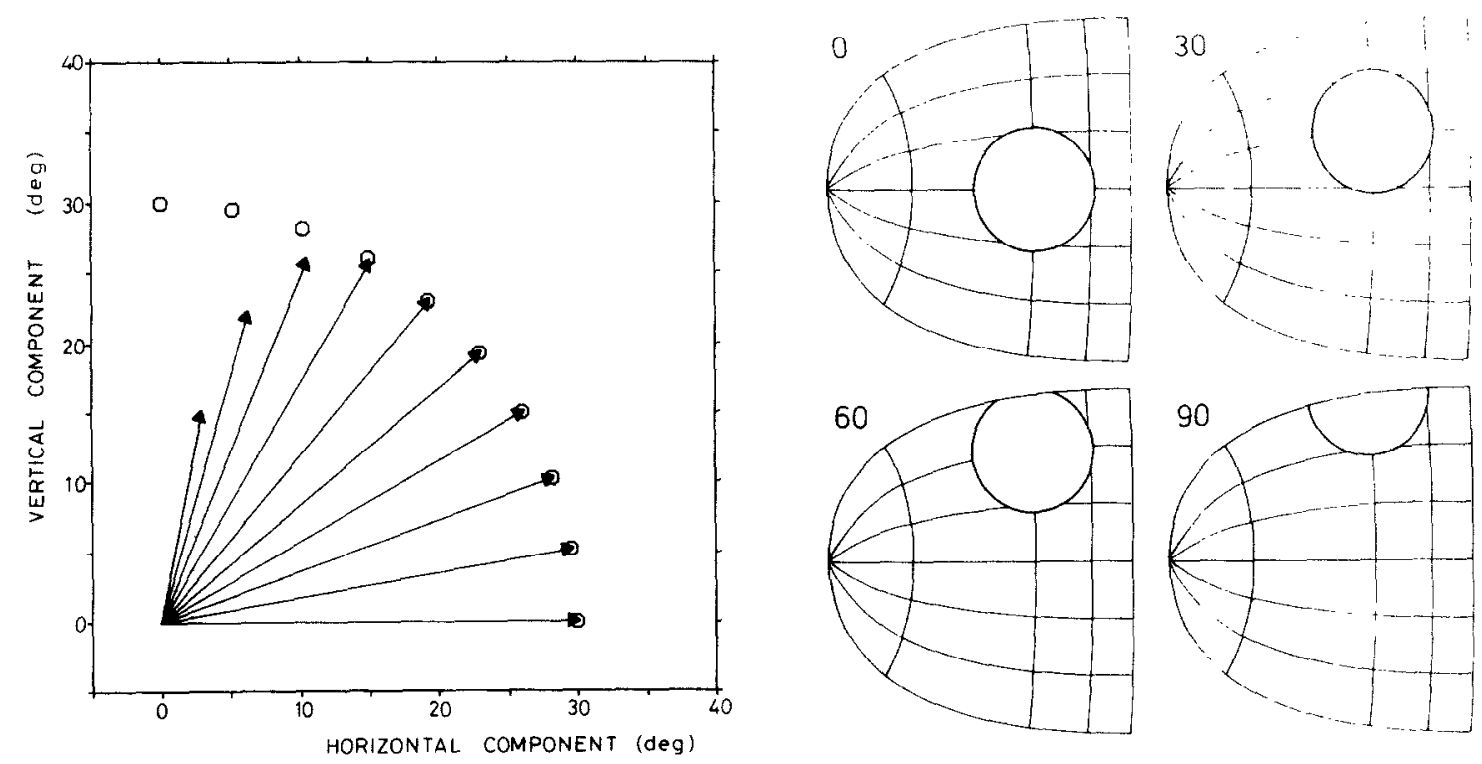

Fig. 7. Border effects in electrical stimulation experiments. The stimulation electrode is moved along the $R=30 \mathrm{deg}$ representation in the SC from the horizontal meridian to the vertical meridian in $10 \mathrm{deg} \Delta \Phi$ steps. The corresponding external world points are denoted by small open circles in the left-hand part of the figure. Note that the model response (arrows) is normometric except near the vertical meridian. The $2 \sigma$ borders of the population activity $(\sigma=0.5 \mathrm{~mm})$ for meridional electrode positions indicated by numbers are shown on the right. The map figures have meridians every $30 \mathrm{deg}$ and lines representing 10 . 30,60 and $100 \mathrm{deg}$ eccentricity.

mechanical limitations, one would expect that saccades with amplitudes of up to $90 \mathrm{deg}$ must be possible if the fixation point is in the far periphery. Since saccades of $90 \mathrm{deg}$ amplitude have indeed been recorded in humans, ${ }^{1}$ the maximal saccade size of about $55 \mathrm{deg}$ in our model is clearly too small. A suggestion for improving the model will be discussed below (section "Center of gravity computation").

Electrical stimulation. The model is able to mimick the important features of responses to SC electrical stimulation (see Results) including the observation that purely vertical saccades cannot be obtained by unilateral SC electrical stimulation. ${ }^{29}$ Saccades obtained by stimulation near the vertical meridian representation are always tilted away from the vertical. Thus, our simulation result that the movement caused by stimulating one colliculus at the $(R=30$, $\Phi=90)$ deg representation site has a direction of $78 \mathrm{deg}$ (not $90 \mathrm{deg}$ ) seems quite realistic (Fig. 7). The other part of the result, that this movement had only a $15 \mathrm{deg}$, rather than a $30 \mathrm{deg}$, vertical component possibly was not. At the moment there is no way of knowing for sure since experiments of the type performed by Schiller and coworkers. ${ }^{32,33}$ where the saccade vector obtained by local electrical SC stimulation was compared with the field location of a nearby SC neuron, have never been done near the collicular borders. Clearly, such experiments need to be undertaken to evaluate the model more fully.

Since the three mapping parameters of the model are already based on electrical stimulation results, model simulation of Robinson's electrical stimulation data $^{29}$ may, at first sight, seem to be a circular exercise. We think it is not, for two reasons. First, Robinson's electrical stimulation data is limited to a part of the SC which does not include its border regions. The mapping equations, fitted to the limited data set, have generalized the mapping over the entire colliculus. The electrical stimulation results of Robinson could only be used to derive the afferent mapping by relying on the finding in Schiller's group $^{32.33}$ that visual fields and movement fields occupy locations corresponding to the saccade vector obtained by local electrical stimulation (Schiller's relationship, see Introduction). The model predicts the existence of edge effects for electrical stimulation sites near the collicular border which imply a violation of Schiller's relationship. Thus the model, partly based on (and compatible with) the validity of Schiller's relationship in a limited region of the SC map, leads directly to the prediction that this relationship cannot be universally valid. Second, an important part of the model [equations (3) through (7)], is not based on electrical stimulation data. Therefore, it is not trivial that the complete model can simulate them.

Effects of lesions. In the model, lesioning a region in the SC map, e.g. around the $(R=20, \Phi=0)$ representation, does not selectively eliminate these saccades from the oculomotor repertoire. In other words, inactivation of a region in the motor colliculus does not cause an equally sharply demarcated saccade defect. This picture also emerges from $\mathrm{SC}$-lesion studies in the monkey. There is evidence that an 


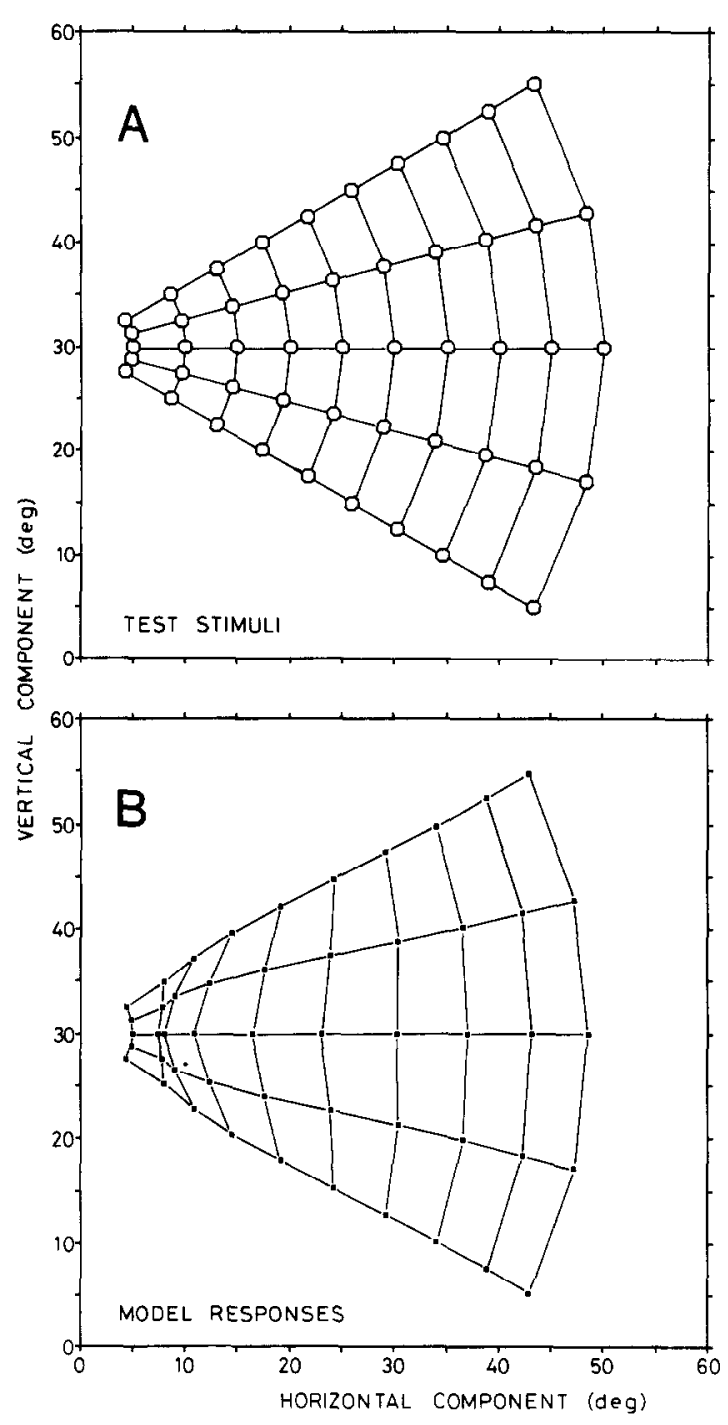

Fig. 8. Model simulation of SC lesion. (A) Location of test stimuli in visual field. (B) Model responses to stimuli shown in (A).

electrolytic lesion ${ }^{47}$ or reversible chemical inactivation of collicular neurons ${ }^{13}$ may, indeed, lead to hypometric responses to stimuli rather than to a complete absence of responses, just as we found in the model simulations. If our model is correct, small dircction errors would also be expected. The actual picture is, of course, more complicated. One complicating factor is that the frontal eye fields also seem to play a role in the visual guidance of saccades., ${ }^{2,34}$ Furthermore, it is known that reversible inactivation of a limited SC region may also cause saccades to become abnormally slow ${ }^{13}$ and this effect cannot be understood from our present model, which would not predict effects on saccade dynamics.

\section{Efferent mechanisms determining saccade metrics}

Considering their potential for generating saccadic eye movements, SC movement cells must have func- tional connections with up, down, right and leftgoing medium-lead saccadic burst cells (MLBs) ${ }^{15,17,43}$ in the reticular formation. There are reasons to suppose that so-called long-lead burst cells (LLBs) are intercalated between the SC movement cells and MLBs. ${ }^{12,27}$ Recently, a model has been presented by Scudder $^{37}$ in which the SC burst signal becomes integrated to create a desired displacement signal at the level of LLBs which drives MLBs during saccades. The challenge of proposing a realistic neural network downstream of the SC becomes quite formidable if the scheme should also account for dynamic properties of saccades ${ }^{37,44}$ but this aspect will not be considered here. The desired displacement signal in Scudder's model, which determines the metrics of the saccade, can be derived from the mean burst rate of SC movement cells [see equations (6) and (7)] if the burst duration of SC neurons is independent of their anatomical location.

The present work allows one to specify the required synaptic weights of each SC movement cell with horizontal and vertical LLBs in Scudder's scheme. Such connections form the anatomical embodiment of the movement potential vector concept introduced above (see Introduction). Their absolute strength corresponds with the size of movement potential vector $\mathbf{P}_{i}$ in equation (6). The relative strengths of the connections with the horizontal and vertical system reflect the direction of this vector. As outlined in the Introduction, these connections would depend entirely on the anatomical position $(u, v)$ of the SC neuron. With an appropriate scaling factor common for all cells, $\alpha$ in equation (6), such a scheme would already perform quite well, despite the complication that for each saccade an entire population of cells is active. As the simulations have shown, however, there are still several problems with such a scheme which seem to require a nonlinear mechanism (see below; section on "Center of gravity computation").

It is conceivable that, in further work, postsynaptic nonlinearities will have to be considered. If such nonlinearities are important, the assumption in equation (6) that $\mathbf{M}$ depends linearly on firing rate $F$, will have to be revised.

\section{Center of gravity computation}

Our model behaves unnaturally when the limited representation in SC map $\left(R_{\lim }=100 \mathrm{deg}\right)$ causes truncation of the population activity profile. This "loss" of neural activity near the collicular border causes maximal saccade size to be as unrealistically low as $55 \mathrm{deg}$ and to drop even further as target eccentricity reaches the limit of the visual field. Another phenomenon, which our vector model cannot explain and which at first sight may seem unrelated, is the averaging phenomenon revealed by electrical double stimulation at two different collicular sites..$^{29}$ In this case the saccade is not the vector sum of the effects created by either stimulus alone, as our model would predict, but a compromise saccade 
which represents a weighted average. Such compromise responses have also been found in response to visual double stimuli, ${ }^{4,8,21,22}$ and, therefore, cannot be dismissed simply as unphysiological.

A possibility to rectify both shortcomings of our model would be to make $\alpha$ in equation (6), which is now fixed, dependent upon the total neural activity in the movement cell population. Suppose, for example, that the synaptic weights with LLBs in Scudder's model (see above) would not be fixed but would be scaled by the total neural activity at the input. This makes the model nonlinear, of course, and requires a summing device which counts the total neural activity (before the spatial weighting) as well as a way of carrying out the scaling operation. A circuit which performs precisely these operations has been proposed to account for gain constancy effects in orientation reactions of the fly in response to relative movement of figures of different widths against a background. ${ }^{28}$ In this circuit a "pool cell" measures the total neural activity in the population of elementary movement detectors and scales their input to the movement control system by presynaptic shunting inhibition. A similar effect can also be obtained by postsynaptic shunting inhibition. ${ }^{6}$ Such a mechanism would in effect carry out a center of gravity computation. $^{8}$

Recently, Tweed and Vilis ${ }^{42}$ have proposed a model of the saccadic system containing a quite different nonlinear mechanism to account for averaging responses to SC double stimulation. Although it remains to be specified more precisely how this mechanism can be realized in a neural wiring diagram, its overall effect is again to scale the effect of individual SC neurons dependent upon the total activity in the population. If such a scaling mechanism exists, the loss of SC movement cell activity caused by a small lesion would be less pronounced than our model simulations would lead one to expect. Also the remarkable independence of saccade amplitude from stimulus intensity, noted in electrical stimulation experiments, ${ }^{29}$ would become more understandable. Further evaluation of these ideas will require more work and is beyond the scope of this paper.

\section{Internal feedback loops}

In Robinson's internal feedback model, ${ }^{30,43}$ MLBs are driven by the difference between a signal representing target location relative to the head (desired eye position, $E d$ ) and an efference copy of eye position $\left(E^{\prime}\right)$. If one attempts to find room for the SC in this scheme, a somewhat paradoxical and confusing situation arises.

On the one hand, since nothing resembling the postulated $E d$ or the $E^{\prime}$ signals is suggested in the known properties of collicular neurons, the idea of an $E d-E^{\prime}$ subtraction stage is not supported by presentday knowledge of collicular neurophysiology. The data available are compatible with the idea that the SC carries only static desired displacement informa- tion, ${ }^{9}$ as we assumed in our vector-summation model. Thus, unlike the MLBs, the SC does not seem to contain dynamic motor error information. In other words, despite the strong evidence for the use of internal feedback in the saccadic system, there is no reason to assume that internal feedback affects the active locus of SC movement cell activity during the saccade. This seems also true when a saccade is made to an auditory target in the dark, a task where target location is likely to be coded in head coordinates. In this case collicular movement cells are recruited when the difference between eye position and target location calls for a saccade corresponding to their optimal saccade vector. ${ }^{14}$ These data suggest that if $E d-E^{\prime}$ subtraction exists, it must occur upstream of the motor colliculus.

On the other hand, it has been shown, in support of Robinson's model, that monkeys trained to make a saccade to a flashed target, in darkness, are able to correct for the eye position disturbance created by collicular electrical stimulation. ${ }^{40}$ Collicular burst neurons have been shown to be involved in the generation of the correction saccade necessitated by the electrically elicited saccade. ${ }^{41}$ The preliminary conclusion suggested by these behavioural and electrophysiological experiments is that there is a need for comparison of $E d$ and $E^{\prime}$ information at least under certain conditions and that the $S C$. receives the outcome of this process. $^{18}$ Furthermore, the difference signal may be spatially coded, rather than in a temporally coded component-related format.

If these views are correct, the total system may have several feedback loops. ${ }^{12}$ First, componentrelated eye velocity feedback signals may be responsible for the tight relation between MLB rate and component motor error. ${ }^{43}$ Second, eye position feedback signals which are compared with the putative $E d$ signal may provide the $\mathrm{SC}$ with information on the desired saccade vector. Our model is consistent with this idea.

\section{Coordinate systems}

The transformation of neural activity in the SC motor map into a saccadic eye movement takes place in a sequence of systems with different frames of reference.

To begin with the SC motor map is twodimensional while, at least in theory, the eye ball has three degrees of freedom. According to Listing's law, however, eye torsion is specified for each horizontal-vertical fixation position of the eye so that, to the extent that this law is valid, there would be only two degrees of freedom. ${ }^{3}$ Whether or not the specification of a three-dimensional movement from a two-dimensional array of neurons presents a problem of indeterminacy in the case of saccades cannot be decided at present. To resolve this uncertainty, Robinson's electrical stimulation study of the SC should be repeated with special emphasis on the analysis of torsion eye movements. In addition, it 


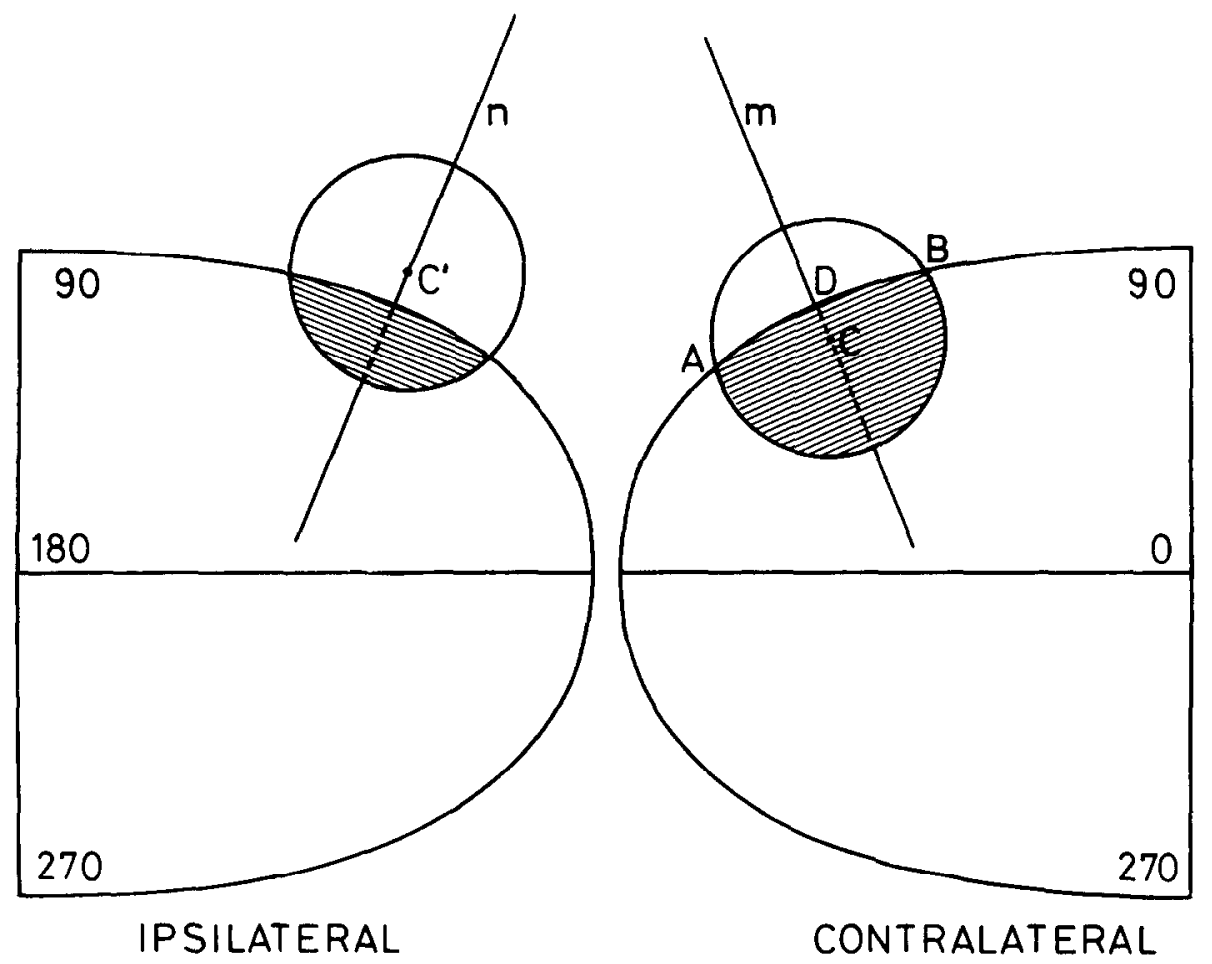

Fig. A1. Procedure to determine movement-cell activity in each colliculus. Shaded regions represent active areas in contralateral and ipsilateral colliculus.

would be useful to know more about the validity of Listing's law for visually elicited saccades.

A further point of interest concerns the assumption of an orthogonal Cartesian coordinate system downstream of the SC which allows simple vectorial summation of the individual movement tendencies, generated by elements in the ensemble, to produce the overall effect [see equation (7)]. Although we recognize that the assumption of orthogonality is not trivial, we do not consider it to be an unrealistic approximation. The assumption is not trivial because there are reasons to think that the muscle system is non-orthogonal. ${ }^{24,25,31}$ Nevertheless, there is evidence from studies of MLBs that, at a premotor level, the saccadic system is organized in a Cartesian coordinate system. ${ }^{9}$ If future work would show that the assumption of orthogonality reflects a too simplistic approach, more sophisticated analyses, able to cope with non-orthogonal systems, will have to be implemented. ${ }^{7,24,25,26,31}$

\section{APPENDIX}

The procedure used to determine the movement cell activity in each colliculus for target positions near the vertical meridian, is as follows. To begin with, the point $\left(u_{c}, v_{c}\right)$ in the contralateral colliculus, indicated by $\mathrm{C}$ in Fig. $\mathrm{Al}$, is determined using equations (1) and (2). The activity profile in this colliculus is found by truncating the Gaussian function [equation (3)] beyond the vertical meridian representation (Fig. A1).

As a preliminary step towards determining the activity profile in the other colliculus, the point on the vertical meridian representation which is closest to $\mathrm{C}$ is found by constructing a line $\mathrm{m}$ which is perpendicular to $\mathrm{AB}$ ( $\mathrm{A}$ and $\mathrm{B}$ are the intersection points between the $2 \sigma$ circle and the vertical meridian representation).

Finally, an equivalent line $\mathrm{n}$ is constructed across the common vertical meridian representation in the ipsilateral colliculus (Fig. A1) and a $2 \sigma$ circle is drawn around the point $\mathrm{C}^{\prime}$ located on $\mathrm{n}$ at a distance $\mathrm{CD}$ beyond the collicular border. The Gaussian activity profile is again truncated beyond the vertical meridian representation.

Acknowledgements - This study was partially supported by the Netherlands Organization for the Advancement of Pure Research (ZWO). We are indebted to Peter Penders for advice and to Marianne Nieuwenhuizen and Astrid van Alst for editorial work. We thank Fenno Ottes and Jos Eggermont for helpful discussions.

\section{REFERENCES}

1. Baloh R. W., Sills A. W., Kumley W. E. and Honrubia V. (1975) Quantitative measurement of saccade amplitude, duration, and velocity. Neurology 25, 1065-1070. 
2. Bruce C. J. and Goldberg M. E. (1985) Primate frontal eye fields. I. Single neurons discharging before saccades. $J$. Neurophysiol. 53, 603-635.

3. Carpenter R. H. S. (1977) Movements of the Eyes. Pion, London.

4. Coren S. and Hoenig P. (1972) Effect of non-target stimuli upon length of voluntary saccades. Percept. Mott. Skills 34, 499-508.

5. Cynader M. and Berman N. (1972) Receptive-field organization of monkey superior colliculus. J. Neurophysiol. 35, $187-201$.

6. Egelhaaf M. (1985) On the neuronal basis of figure-ground discrimination by relative motion in the visual system of the fly. I. Behavioural constraints imposed on the neuronal network and the role of the optomotor system. Biol. Cybern. 52, $123-140$.

7. Ezure K. and Graf W. (1984) A quantitative analysis of the spatial organization of the vestibulo-ocular reflexes in lateral and frontal-eyed animals-II. Neuronal networks underlying vestibulo-oculomotor coordination. Neuroscience 12, 95-109.

8. Findlay J. M. (1982) Global visual processing for saccadic eye movements. Vision Res. 22, 1033-1045.

9. Fuchs A. F., Kaneko C. R. S. and Scudder C. A. (1985) Brainstem control of saccadic eye movements. Ann. Ket Neurosci. 8, 307-337.

10. Georgopoulos A. P., Caminiti R., Kalaska J. F. and Massey J. T. (1983) Spatial coding of movement: a hypothesis concerning the coding of movement direction by motor cortical populations. Exp. Brain Res. Supplement 7, 327-336.

11. Georgopoulos A. P., Kalaska J. F., Crutcher M. D., Caminiti R. and Massey J. T. (1984) The representation of movement direction in the motor cortex: single cell and population studies. In Dynamic Aspects of Neocortical Function (eds Fdelman G. M., Gall W. E. and Cowan W. M.), pp. 501-524. Wiley, New York.

12. Hepp K. and Henn V. (1983) Spatio-temporal recoding of rapid eye movement signals in the monkey paramedian pontine reticular formation (PPRF). Exp. Brain Res. 52, 105-120.

13. Hikosaka O. and Wurtz R. H. (1985) Modification of saccadic eye movements by GABA-related substances. I. Effect of muscimol and bicuculline in monkey superior colliculus. J. Neurophysiol. 53, 266-291.

14. Jay M. F. and Sparks D. L. (1984) Auditory receptive fields in primate superior colliculus shift with changes in eye position. Nature 309, 345-347.

15. Keller E. L. (1974) Participation of medial pontine reticular formation in eye movement generation in monkey. $J$ Neurophysiol. 37, 316-332.

16. Keller E. L. (1980) Oculomotor specificity within subdivisions of the brain stem reticular formation. In The Reticular Formation Revisited. (eds Hobson J. A. and Brazier M. A. B.), pp. 227.240 . Raven Press, New York.

17. Luschei E. S. and Fuchs A. F. (1972) Activity of brain stem neurons during eye movements of alert monkeys. $J$. Neurophysiol. 35, 445-461.

18. Mays L. E. and Sparks D. L. (1980) Dissociation of visual and saccade-related responses in superior colliculus neurons. J. Neurophysiol. 43, 207-232.

19. Mcllwain J. T. (1976) Large receptive fields and spatial transformations in the visual system. In Neurophysiology $I I$. (ed. Porter R.), Vol. 10, pp. 223-248. Univ. Park Press, Baltimore.

20. McIlwain J. T. (1982) Lateral spread of neural excitation during microstimulation in intermediate gray layer of cat's superior colliculus. $J$. Neurophysiol. 47, 167-178.

21. Ottes F. P., Van Gisbergen J. A. M. and Eggermont J. J. (1984) Metrics of saccade responses to visual double stimuli: two different modes. Vision Res. 24, 1169-1179.

22. Ottes F. P., Van Gisbergen J. A. M. and Eggermont J. J. (1985) Latency dependence of colour-based target vs nontarget discrimination by the saccadic system. Vision Res. 25, 849-862.

23. Ottes F. P., Van Gisbergen J. A. M. and Eggermont J. J. (1986) Visuomotor fields of the superior colliculus: a quantitative model. Vision Res. 26, 857-873.

24. Ostriker G., Pellionisz A. and Llinas R. (1985) Tensorial computer model of gaze-I. Oculomotor activity is expressed in non-orthogonal natural coordinates. Neuroscience 14, 483-500.

25. Pellionisz A. (1985) Tensorial aspects of the multidimensional approach to the vestibulo-oculomotor reffex and gaze. In Adaptive Mechanisms in Gaze Control: Facts and Theories (eds Berthoz A. and Melvill Jones G.), pp. $281-296$. Elsevier, Amsterdam.

26. Pellionisz A. and Llinas R. (1980) Tensorial approach to the geometry of brain function: cerebellar coordination via a metric tensor. Neuroscience 5, 1125-1136.

27. Raybourn M. S. and Keller E. L. (1977) Colliculoreticular organization in primate oculomotor system. J. Neurophysiol. 40, 861878

28. Reichardt W., Poggio T. and Hausen K. (1983) Figure-ground discrimination by relative movement in the visual system of the fly. Part II: Towards the neural circuitry. Biol. Cybern. 46, (suppl.) 1-30.

29. Robinson D. A. (1972) Eye movements evoked by collicular stimulation in the alert monkey. Vision Res. 12, $1795-1808$.

30. Robinson D. A. (1975) Oculomotor control signals. In Basic Mechanisms of Ocular Motility and their Clinical Implications (eds Lennerstrand G. and Bach-y-Rita P.), pp. 337-374. Pergamon Press, Oxford.

31. Robinson D. A. (1982) The use of matrices in analyzing the three-dimensional behavior of the vestibulo-ocular reflex. Biol. Cybern. 46, 53-66.

32. Schiller P. H. and Koerner F. (1971) Discharge characteristics of single units in superior colliculus of the alert rhesus monkey. J. Neurophysiol. 34, 920-936.

33. Schiller P. H. and Stryker M. (1972) Single-unit recording and stimulation in superior colliculus of the alcrt rhesus monkey. J. Neurophysiol. 35, 915-924.

34. Schiller P. H., True S. D. and Conway J. L. (1980) Deficits in eye movements following frontal eye field and superior colliculus ablations. J. Neurophysiol. 44, 1175-1189.

35. Schiller P. H. (1984) The superior colliculus and visual function. In Handbook of Physiology. Section 1: The Nervous System, Vol. III, pp. 457-505. American Physiological Society, Bethesda.

36. Schwartz E. L. (1980) Computational anatomy and functional architecture of striate cortex: A spatial mapping approach to perceptual coding. Vision Res. 20, 645 669.

37. Scudder C. A. (1985) Functional identification and characterisation of saccadic inhibitory burst neurons in the behaving monkey. Thesis, University of Washington, Seattle. 
38. Sparks D. L., Holland R. and Guthrie B. L. (1976) Size and distribution of movement fields in the monkey superior colliculus. Brain Res. 113, $21-34$.

39. Sparks D. L. and Mays L. E. (1981) The role of the superior colliculus in the control of saccadic eye movements: A current perspective. In Progress in Oculomotor Research (eds Fuchs A. F. and Becker W.), pp. 137-144. Elsevier North-Holland, Amsterdam.

40. Sparks D. L. and Mays L. E. (1983) Spatial localization of saccade targets. I. Compensation for stimulation induced perturbations in eye position. $J$. Neurophysiol. 49, 45-63.

41. Sparks D. L. and Porter J. D. (1983) Spatial localization of saccade targets. II. Activity of superior colliculus neurons preceding compensatory saccades. $J$. Neurophysiol. $49,64-74$.

42. Tweed D. and Vilis T. (1985) A two dimensional model for saccade generation. Biol. Cybern. 52, $219-227$.

43. Van Gisbergen J. A. M., Robinson D. A. and Gielen S. (1981) A quantitative analysis of generation of saccadic eye movements by burst neurons. $J$. Neurophysiol. 45, 417-442.

44. Van Gisbergen J. A. M., Van Opstal A. J. and Schoenmakers J. J. M. (1985) Experimental test of two models for the generation of oblique saccades. Exp. Brain Res. 57, 321-336.

45. Wurtz R. H. and Albano J. E. (1980) Visual-motor function of the primate superior colliculus. Ann. Rev. Neurosci. $3,189-226$.

46. Wurtz R. H. and Goldberg M. E. (1972) Activity of superior colliculus in behaving monkey. III. Cells discharging before eye movements. J. Neurophysiol. 35, 575-586.

47. Wurtz R. H. and Goldberg M. E. (1972) Activity of superior colliculus in behaving monkey. IV. Effects of lesions on eye movements. J. Neurophysiol. 35, 587-596. 